\title{
Une nouvelle étude sur l'effet des faibles doses
}

\author{
M. DOUSSET* et H. JAMMET** \\ (Manuscrit reçu le 11 décembre 1985)
}

\begin{abstract}
RÉSUMÉ
Cette étude [2], dirigée par le professeur ROSE de la London School of hygiene and Tropical Medicine, analyse les causes de mortalité parmi 39546 employés de certains établissements de l'UKAEA, suivis de 1946 à 1978. Les résultats peuvent se résumer en trois points:

1. La mortalité générale et la mortalité par affection maligne sont inférieures à celles de la population de l'Angleterre et du Pays de Galles $(74 \%$ et $79 \%$ ). De ce point de vue il n'y a pas de différence majeure entre les sujets surveillés pour l'exposition aux rayonnements et les autres.

2. Pour le personnel surveillé, la seule cause de décès pour laquelle il y aurait une corrélation statistiquement significative avec l'exposition aux rayonnements est le cancer de la prostate; ces cas sont particulièrement nombreux chez les sujets ayant reçu plus de $0,05 \mathrm{~Sv}$ ( 5 rems) et faisant l'objet d'une surveillance radiotoxicologique pour le tritium. Une telle corrélation ne se retrouve dans aucune autre étude épidémiologique sur des sujets exposés à de faibles doses, en particulier celle sur les travailleurs du Centre de Hanford (USA); et, réciproquement, ni la mortalité par myélome multiple ni celle par cancer du pancréas ne se retrouvent ici. Ces faits plaident pour une certaine prudence dans l'interprétation de l'ensemble de ces résultats.
\end{abstract}

3. La recherche d'une représentation linéaire pour rendre compte des variations de la mortalité par leucémie et par cancer avec l'exposition conduit à des droites dont la pente est environ trois fois supérieure à celle des droites adoptées par la CIPR; mais les marges d'incertitude $(-2,7+12,4$ et $-22+52,2)$ sont tellement grandes que ces résultats sont compatibles avec un effet nul (pente 0 ) et même avec un certain effet bénéfique (pente négative). Ils sont donc parfaitement compatibles avec les estimations de la CIPR.

La synthèse des deux grandes études sur l'exposition professionnelle à faibles doses (UKAEA et Hanford) a déjà été tentée et suggère une relation dose-réponse très proche de celle de la CIPR.

* 7, rue de la Gruerie, 91190 Gif-sur-Yvette.

** Commissariat à l'énergie atomique, BP 6, 92265 Fontenay-aux-Roses Cedex. 


\section{ABSTRACT}

A study conducted by prof. ROSE [2] has investigated mortality among 39540 employees of the UKAEA, from 1946 to 1978.

The three main points are:

1. General mortality and mortality from malignant diseases are lower than in the population of England and Wales (74 and 79 per cent respectively), thus showing no major difference between workers monitored for exposure to radiation and other workers.

2. For monitored workers, the only death cause for which there is a statistically significant correlation with radiation exposure is prostate cancer; there are many cases especially in workers with doses exceeding $0.05 \mathrm{~Sv}$ ( 5 rem) and monitored for tritium. Such a correlation has never been found in any other epidemiologic survey of workers exposed to low-level doses, Hanford (USA) workers especially; conversely, mortality from either multiple myeloma or pancreas cancer is not found here. These facts plead for a cautious interpretation of the results as a whole.

3. A linear representation of the variations of leukemia and cancer mortality vs exposure results in lines, the slopes of which are 3 times higher than those of the lines adopted by ICRP; however, the 95 per cent confidence intervals $(-2.7+12.4$ and $-22+52.5)$ are such that the results are compatible with a null effect (slope 0 ) and even with a benefic effect (negative slope). They are therefore compatible with ICRP estimations.

A recent attempt to evaluate the two main investigations on low-dose occupational exposures (UKAEA and Hanford) suggests a dose-response relationship very near that of ICRP.

Dans son numéro du 17 août 1985 , le British Medical Journal a publié une étude du prof. Geoffroy ROSE et de ses collaborateurs [2] sur les causes de mortalité parmi 39546 personnes qui ont travaillé entre le $1^{\text {er }}$ janvier 1946 et le 31 décembre 1979 dans certains établissements de I'United Kingdom Atomic Energy Authority (UKAEA); ces sujets ont été suivis sur une durée moyenne de 16 ans. Les caractéristiques de cette population sont données dans le tableau I.

TABLEAU ।

Caractéristiques de la population étudiée

\begin{tabular}{|c|c|c|c|c|}
\hline \multirow[b]{2}{*}{ A } & \multirow[b]{2}{*}{$\begin{array}{l}\text { Personnel pour lequel les résultats de } \\
\text { la surveillance radiologique indivi- } \\
\text { duelle ont étè enregistrès }\end{array}$} & Hommes & Femmes & Totaux \\
\hline & & 18759 & 1623 & 20382 \\
\hline \multirow[t]{2}{*}{ B } & $\begin{array}{l}\text { Personnel pour lequel il n'y avait pas } \\
\text { de surveillance radiologique } \\
\text { individuelle }\end{array}$ & 10266 & 8898 & 19164 \\
\hline & & 29025 & 10521 & 39546 \\
\hline
\end{tabular}


L'objectif était de rechercher s'il n'y avait pas une relation entre la mortalité, plus particulièrement la mortalité par cancer, et l'exposition aux rayonnements en vue de comparer les résultats obtenus avec les estimations retenues par la Commission internationale de protection radiologique (CIPR) dans sa publication 26 de 1977 [4]. Compte tenu du nombre de sujets et du temps d'observation, les auteurs étaient bien conscients, avant même que l'étude ne commence, de la capacité limitée de leur analyse et ils précisèrent qu'elle ne pourrait mettre en évidence une différence statistiquement significative que dans le cas oủ la CIPR aurait sous-estimé le risque d'un facteur 20 ou plus. Malgré cette mise en garde, la presse britannique, dès la publication de l'article, se hâta d'affirmer que cette nouvelle étude mettait en cause les évaluations de la CIPR et que le risque aux faibles doses que cette dernière avait retenu en 1977 devait être multipliè par 15 , ce qui conduirait à réduire d'autant les valeurs des limites de doses recommandées par la Commission. Voyons ce qu'il en est en procédant à une analyse de la publication de $\mathrm{G}$. Rose et en rapprochant les résultats obtenus de ceux qui ont été antérieurement publiés dans des travaux analogues sur les causes de mortalité chez des sujets professionnellement exposés aux rayonnements.

Le travail des auteurs britanniques comporte trois stades complémentaires qui sont développés successivement:

- une comparaison de la mortalité des employés de I'UKAEA avec celle de la population nationale (Angleterre et Pays de Galles);

- une recherche de corrélations entre la mortalité par différentes affections malignes et le niveau d'exposition chez les employés qui ont fait l'objet d'une surveillance radiologique individuelle dont les résultats ont été enregistrés (pour simplifier le texte et les tableaux qui vont suivre, ces employés seront désignés ici comme appartenant au personnel de "catégorie A" par opposition à ceux qui n'ont pas fait l'objet d'une telle surveillance: personnel de "catégorie $\left.B^{\prime \prime}\right)$;

- une recherche de relation quantitative dose-réponse pour la mortalité par leucémie et pour la mortalité par l'ensemble des cancers.

\section{COMPARAISON AVEC LA MORTALITÉ NATIONALE}

Pour comparer la mortalité dans un groupe donné de personnes avec la mortalité d'une population témoin, on calcule le nombre de décès qui aurait été trouvé dans ce groupe si ce dernier avait, pour chaque sexe, les mêmes caractéristiques que la population témoin. Ici, la population témoin est la population nationale (Angleterre et Pays de Galles) et les classes d'âge retenues sont au nombre de 12, entre 15 et 74 ans. Le nombre ainsi calculé est appelé "nombre attendu" et la comparaison se fait, en particulier dans les pays anglo-saxons, en effectuant le rapport (exprimé en pour cent) du nombre de décès observé au nombre de décès attendu: ce pourcentage est appelé en anglais: "standardised mortality ratio" ou "SMR".

La valeur du SMR n'a pas, en elle-même, une signification absolue et il est nécessaire d'indiquer le nombre de cas attendu (ou observé) pour pouvoir calculer la probabilité que le résultat trouvé soit dû au hasard; on comprend facilement, en effet, que si le nombre de cas attendu est faible $(0,1$ par exemple) il suffit d'observer 1 cas pour avoir un SMR de 1000, alors que la loi de Poisson indique qu'il y a 9,5 chances sur 100 d'observer par hasard 1 cas ou plus avec une moyenne de 0,1 . Or, il est convenu que l'excès constaté ne commence à 
avoir une signification statistique que si la probabilité de trouver par hasard le nombre observé ou plus est inférieure à 0,025; par convention également, cet excès devient hautement significatif lorsque la probabilité de trouver par hasard le nombre observé ou plus est inférieure à 0,01 .

Pour présenter les résultats obtenus par le $\mathrm{Pr}$. G. ROSE et ses collaborateurs dans cette comparaison avec la population nationale, il y a lieu de distinguer selon les différentes causes de mortalité et la façon de les regrouper:

- mortalité quelle qu'en soit la cause (mortalité générale):

- mortalité par affection maligne quelle qu'elle soit;

- mortalité par affection maligne de différentes localisations.

Dans chaque cas, il faudra également distinguer entre les 2 catégories ( $A$ et B) de personnel telles qu'elles ont été définies plus haut.

\subsection{Mortalité générale (tableau II)}

Les résultats concernant la mortalité générale font l'objet de la première partie du tableau II. On peut constater que la mortalité générale aussi bien pour le personnel de la classe $A$ que pour celui de la classe $B$ est inférieure, d'une façon hautement significative, à celle de la population nationale. Ce résultat est conforme à ce que l'on pouvait attendre pour le personnel d'une grande entreprise composé de sujets sélectionnés à l'embauche sur des critères de santé, étroitement suivi du point de vue médical, et de statut socio-économique supérieur à la moyenne. Les anglo-saxons appellent ce phénomène le "healthy working effect".

\subsection{Mortalité par affection maligne (tableau II)}

TABLEAU ॥

SMR pour la mortalité générale et la mortalité par affection maligne

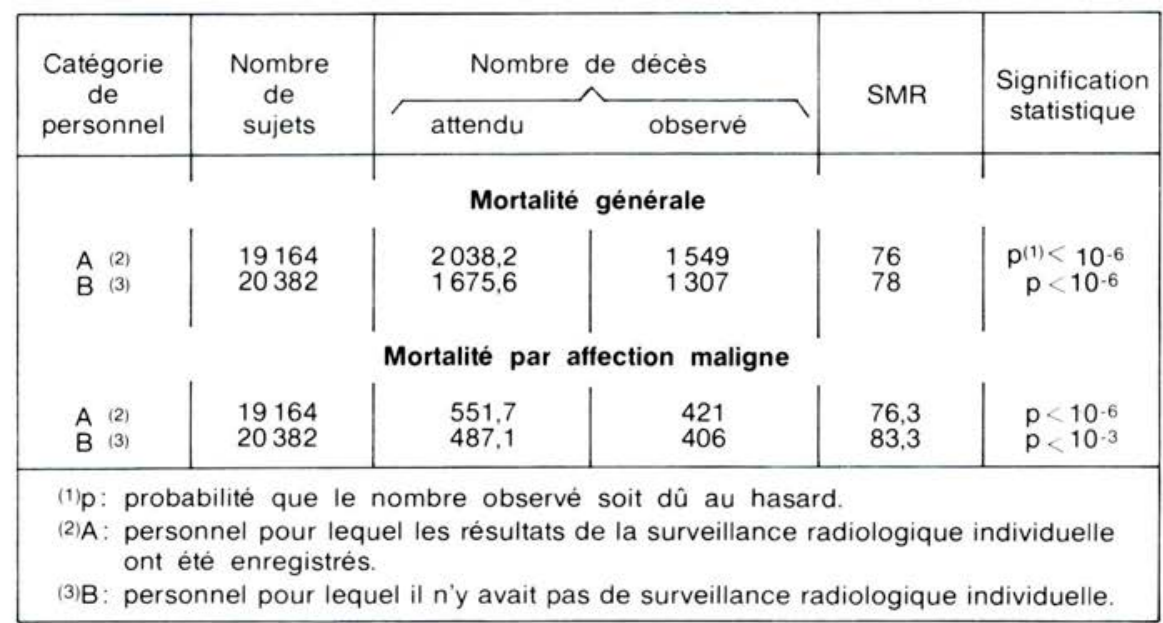


La deuxième partie du tableau II reprend les mêmes éléments : nombre attendu, nombre observé et SMR pour la mortalité par affection maligne quelle qu'elle soit. En général, le "healthy working effect" est moins efficace pour la mortalité par affection maligne que pour la mortalité générale et on explique cette différence par la difficulté de dépister les sujets à risque à l'embauche (ou à l'affectation à un poste exposé). Ici, il n'en est rien et on peut constater qu'au moins pour le personnel de classe A, l'efficacité de "l'effet" est le même que pour la mortalité générale.

\subsection{Mortalité par différents types d'affection maligne (tableau III)}

Pour la mortalité par différents types d'affection maligne, les auteurs analysent 18 localisations particulières. Pour 11 d'entre elles, les SMR pour le personnel de catégorie A sont inférieurs à 100. Pour les autres, le tableau III donne le nombre de cas attendu, le nombre de cas observé, le SMR et la signification statistique : aucune différence n'est statistiquement significative.

TABLEAU III

Affections malignes pour lesquelles les SMR sont supérieurs à 100 pour le personnel de catégorie $A$

\begin{tabular}{|c|c|c|c|c|c|}
\hline \multirow{2}{*}{ Cause de mortalité } & \multirow{2}{*}{$\begin{array}{l}\text { Catégorie } \\
\text { de } \\
\text { personnel }\end{array}$} & \multicolumn{2}{|c|}{ Nombre de décès } & \multirow{2}{*}{ SMR } & \multirow{2}{*}{$\begin{array}{l}\text { Signification } \\
\text { statistique }\end{array}$} \\
\hline & & attendu & observé & & \\
\hline \multirow{2}{*}{$\begin{array}{l}\text { Cancer des tissus } \\
\text { conjonctifs et } \\
\text { des tissus mous }\end{array}$} & A & 1,66 & 2 & 120 & $p>0,25$ \\
\hline & B & 0,96 & 1 & 104 & $p>0,36$ \\
\hline \multirow{2}{*}{$\begin{array}{l}\text { Cancer de l'utérus } \\
\text { et des ovaires }\end{array}$} & A & 4,92 & 9 & 183 & $p \simeq 0,06$ \\
\hline & B & 27.94 & 19 & 68 & \\
\hline \multirow{2}{*}{$\begin{array}{l}\text { Cancer de } \\
\text { la prostate }\end{array}$} & A & 16,52 & 19 & 115 & $p>0,22$ \\
\hline & B & 11,39 & 9 & 79 & \\
\hline \multirow{2}{*}{$\begin{array}{l}\text { Cancer } \\
\text { du testicule }\end{array}$} & A & 4,23 & 6 & 142 & $p>0,18$ \\
\hline & B & 2,31 & 4 & 173 & $p>0,13$ \\
\hline \multirow{2}{*}{$\begin{array}{l}\text { Cancer de } \\
\text { la thyroide }\end{array}$} & A & 1,04 & 2 & 192 & $p>0,26$ \\
\hline & B & 0,63 & 1 & 158 & $p>0,45$ \\
\hline \multirow{2}{*}{$\begin{array}{l}\text { Lymphomes sauf } \\
\text { maladie } \\
\text { de Hodgkin }\end{array}$} & A & 10,49 & 12 & 114 & $p>0,30$ \\
\hline & B & 8,20 & 8 & 98 & \\
\hline \multirow[t]{2}{*}{ Leucémie } & A & 15,36 & 18 & 117 & $p>0,23$ \\
\hline & B & 13,08 & 17 & 130 & $p>0,16$ \\
\hline
\end{tabular}

Pour la signification des symboles A, B et p voir tableau II. 
Le nombre de décès attendu a été calculé à partir des données du tableau III de la publication de G. ROSE. Pour les cancers des tissus conjonctifs et des tissus mous, de même que pour les cancers de la thyroïde, l'absence de cas chez les femmes et le manque d'indications sur le nombre de cas attendu nous ont contraints à ne tenir compte que des données concernant la population masculine. Les auteurs notent qu'il n'y a pas de différence majeure entre le personnel de la classe A et celui de la classe B à l'exception, chez les femmes, de la mortalité par cancers de l'utérus et des ovaires pour lesquels, dans la classe A, la probabilité d'observer par hasard 9 cas ou plus avec une moyenne de 4,92 est proche de 0,06.

\subsection{Evolution des SMR en fonction de la durée d'emploi à l'UKAEA}

Les auteurs ont recherché comment variaient les SMR en fonction de la durée d'emploi des sujets dans les établissements de I'UKAEA. Les SMR pour la mortalité générale et pour la mortalité par leucémie croissent avec la durée de l'emploi pour les sujets de la catégorie A, alors qu'ils décroissent pour les sujets de la catégorie B. Le tableau IV explicite les différentes valeurs qui, pour la leucémie, mettent en évidence ces deux tendances qui sont significativement différentes avec une probabilité que cela soit dû au hasard inférieure à 0,05.

TABLEAU IV

SMR pour la mortalité par leucémie en fonction de la durée d'emploi à l'UKAEA

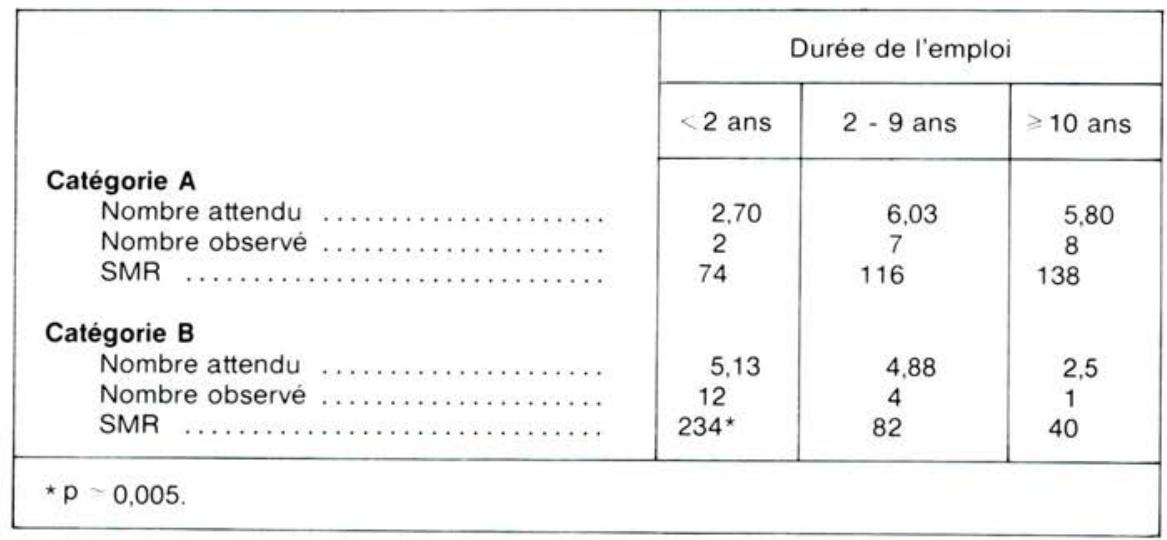

Pour la signification des symboles A, B et $\mathrm{p}$ voir tableau II.

Cependant, le groupe des employés qui appartiennent à la catégorie $B$ et qui sont restés moins de 2 ans dans leur emploi à l'UKAEA (essentiellement du personnel administratif) présente des caractéristiques anormales avec un SMR pour la mortalité par leucémie de 234 hautement significatif. Les auteurs soulignent cette anomalie et envisagent un certain nombre d'hypothèses pour tenter de l'expliquer; mais ce ne sont que des hypothèses et, par suite, ils restent très prudents sur l'inter- 
prétation à donner à ces deux tendances opposées: en attendant qu'on en sache davantage sur les raisons de cette différence, qui peut être due à des facteurs autres que l'exposition aux rayonnements, les comparaisons entre les deux catégories de personnel sont strictement sans valeur: "strictly not valid".

\section{CORRÉLATION ENTRE MORTALITÉ ET NIVEAU D'EXPOSITION POUR LE PERSONNEL DE CATÉGORIE A}

\subsection{Données de base}

Pour cette recherche, le personnel de catégorie $A$ a été réparti en 5 classes selon le niveau de dose cumulée pendant la vie professionnelle. II s'agit de la somme des doses mensuelles résultant de la lecture des dosimètres individuels, les doses inférieures au seuil de sensibilité du dosimètre étant considérées comme nulles. L'exposition aux sources naturelles de rayonnements et l'exposition au cours d'examens ou traitements médicaux n'ont pas été prises en compte.

En règle générale, pour les sujets exposés au risque de contamination interne et qui ont fait l'objet d'une surveillance radiotoxicologique, les doses engagées éventuelles n'ont pas été comptées dans l'évaluation de la dose cumulée, à l'exception, à partir de 1977, de celles dues à l'incorporation de tritium à Harwell. Le tableau $V$ précise les 5 classes d'exposition retenues, le nombre de personnes de catégorie $\mathrm{A}$ et la dose moyenne individuelle correspondante.

\section{TABLEAU V}

Distribution en $\mathbf{5}$ classes du personnel de catégorie A

\begin{tabular}{|c|c|c|}
\hline $\begin{array}{c}\text { Classes d'exposition } \\
\text { (doses en } 10^{-2} \text { Sv ou rem) }\end{array}$ & Nombre de sujets & $\begin{array}{c}\text { Dose moyenne } \\
\text { (en 10 }{ }^{-2} \text { Sv ou rem) }\end{array}$ \\
\hline $0 \leqslant D<1$ & 12423 & 0,16 \\
$1 \leq D<2$ & 2214 & 1,40 \\
$2 \leqslant D<5$ & 2527 & 3,05 \\
$5 \leqslant D<10$ & 1459 & 6,87 \\
$D \geqslant 10$ & 1759 & 17,3 \\
\hline
\end{tabular}

Dans un premier temps, les auteurs ont pris en compte la mortalité à tous âges et ont procédé à une analyse interne ne faisant intervenir que le personnel de l'UKAEA retenu pour cette étude. Le nombre de cas attendu dans chaque classe d'exposition a donc été calculé en prenant l'ensemble de ce personnel comme population témoin et en procédant aux ajustements classiques pour l'âge, le sexe, la période d'observation mais aussi pour le niveau socio-professionnel du personnel et pour les particularités de certains établissements de l'UKAEA. Le nombre de cas attendu ainsi calculé diffère donc de celui qu'on a obtenu en prenant la population de l'Angleterre et du Pays de Galles comme population témoin pour calculer les SMR. 


\subsection{Résultats}

Pour la mortalité générale et pour les affections malignes concernant 5 localisations (estomac, pancréas, poumon, sein et système nerveux central), on obtient des corrélations négatives avec valeurs non significatives du test de tendance linéaire.

Ce n'est que pour le cancer de la prostate qu'on trouve une corrélation positive avec valeur significative du test de tendance linéaire $(p<0,001)$. Le tableau VI donne tous les éléments quantitatifs de jugement qui conduisent à affirmer cette forte corrélation. Lorsqu'on reprend les données de base en tenant compte d'un temps de latence de 15 ans, c'est-à-dire en éliminant tous les cas survenus dans les 15 premières années qui ont suivi le début de l'exposition professionnelle, presque toutes les corrélations deviennent négatives, mais la mortalité par cancer de la prostate présente toujours une corrélation positive avec les doses, et la valeur du test de tendance $(p<0,01)$ est encore significative.

\section{TABLEAU VI}

\section{Corrélation entre l'exposition et la mortalité par cancer de la prostate}

\begin{tabular}{|l|l|c|c|c|c|}
\hline & \multicolumn{5}{|c|}{ Dose cumulée (en $10^{-2}$ Sv ou rem) } \\
\cline { 2 - 6 } & $\mathrm{D}<\mathbf{1}$ & $1 \leqslant \mathrm{D}<\mathbf{2}$ & $\mathbf{2} \leqslant \mathrm{D}<\mathbf{5}$ & $\mathbf{5} \leqslant \mathrm{D}<\mathbf{1 0}$ & $\mathrm{D} \geqslant 10$ \\
\cline { 2 - 7 } & 15,71 & 2,86 & 2,63 & 1,57 & 2,16 \\
Nombre de cas attendu (A) & 11 & 1 & 3 & 3 & 7 \\
Nombre de cas observé (O) & 0,70 & 0,35 & 1,14 & 1,91 & 3,24 \\
Rapport O/A & 0,001 & \\
\hline
\end{tabular}

TABLEAU VII

SMR pour la mortalité par cancer de la prostate selon la dose cumulée et la surveillance du tritium

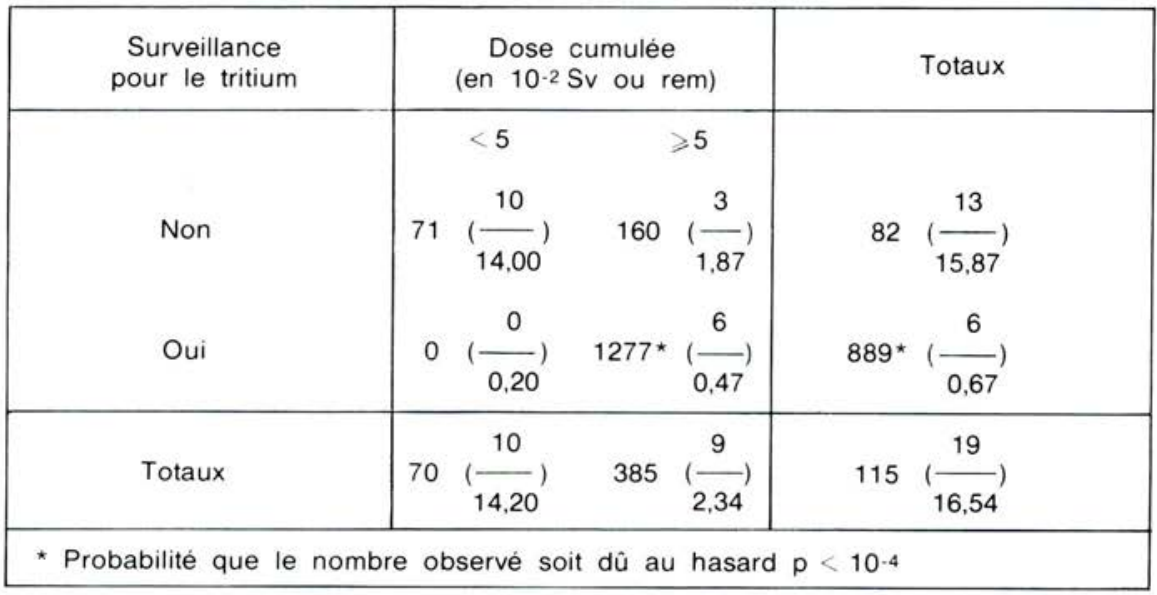


On obtient des résultats analogues en évaluant les SMR avec les 12 tranches d'âge et la population nationale comme population témoin. On trouve alors 19 cas qui se répartissent tout spécialement parmi les sujets pour lesquels la dose cumulée est supérieure à $0,05 \mathrm{~Sv}$ ( 5 rems) et qui, en outre, font l'objet d'une surveillance radiotoxicologique pour le tritium, comme on peut le constater dans le tableau VII.

\subsection{Discussion des résultats}

\subsubsection{Résultats sur la mortalité par cancer de la prostate}

Ces résultats sont surprenants à plus d'un titre:

2.3.1.1. En 1980, le Comité de l'Académie nationale des sciences (USA) sur les effets biologiques des rayonnements ionisants [5] classait la prostate parmi les tissus pour lesquels les affections malignes radioinduites n'avaient pas été observées (tableau $\mathrm{V}$, appendix A).

2.3.1.2. Ni les études sur les survivants de Hiroshima et de Nagasaki $(1,10) \mathrm{ni}$ celles sur les malades atteints de spondylarthrite ankylosante et soignés par rayons $X[12]$ n'ont montré d'augmentation notable de la mortalité par cancer de la prostate.

2.3.1.3. Dans l'étude menée par E.S. GILBERT et S. MARKS $[8,9]$ sur la mortalité par cancer parmi le personnel, suivi jusqu'en mai 1977, de l'usine de Hanford (USA) aucune tendance d'augmentation avec la dose de la mortalité par cancer de la prostate n'apparaît, comme le montre le tableau VIII. II s'agit là d'une analyse interne analogue à celle menée par G. ROSE pour les employés de I'UKAEA. Cependant, il faut signaler que, dans cette étude, la répartition des 12679 hommes de race blanche, plus spécialement étudiés, en 2 catégories professionnelles - ouvriers specialisés et opérateurs (4853) d'une part et tous les autres (7 826) d'autre part - fait apparaître une différence entre elles dans les SMR relatifs aux affections malignes: 96 pour les premiers, 79 pour les seconds. Cette différence est due à une plus grande mortalité, chez les premiers, par cancer du poumon et cancer de la prostate. Les auteurs ne donnent aucune information quantitative sur cette question; ils pensent que l'exposition aux rayonnements ne joue aucun rôle dans cette différence puisqu'il est établi d'autre part qu'il n'y a pas de corrélation statistiquement significative entre l'exposition et la mortalité aussi bien par cancer de la prostate que par cancer du poumon.

TABLEAU VIII

Corrélation entre l'exposition et la mortalité par cancer de la prostate parmi les hommes de race blanche employés plus de 2 ans à l'usine de Hanford, d'après [9]

\begin{tabular}{|l|c|c|c|c|}
\hline \multirow{2}{*}{} & \multicolumn{4}{|c|}{ Doses cumulées (en 10-2 Sv ou rem) } \\
\cline { 2 - 5 } & $0 \leqslant \mathrm{D}<2$ & $2 \leqslant \mathrm{D}<5$ & $5 \leqslant \mathrm{D}<15$ & $\mathrm{D} \geqslant 15$ \\
\cline { 2 - 5 } Nombre de cas attendu (A) & 24,0 & 4,6 & 3,0 & 2,5 \\
Nombre de cas observé (O) & 26 & 6 & 2 & 0 \\
Rapport O/A & 1,08 & 1,30 & 0,87 & 0 \\
\hline Probabilité que la tendance (négative) soit due au hasard $p>0,97$. & \\
\hline
\end{tabular}


2.3.1.4. On a trouvé dans le personnel du Oak Ridge National Laboratory (ORNL) un certain excès de mortalité par cancer de la prostate [3], mais cet excès n'était pas significatif et on n'a pas constaté de corrélation avec l'exposition aux rayonnements.

2.3.1.5. Enfin, dans aucune des études précédemment citées, il n'est mentionné que les sujets morts d'un cancer de la prostate, ou certains d'entre eux, aient été exposés au tritium. D'autre part, le tritium ne se concentre pas dans la prostate et les doses que sa présence dans l'organisme aurait pu entraîner pour les sujets exposés (doses qui n'ont pas été prises en compte, sauf à Harwell à partir de 1977) semblent avoir été faibles d'après les informations non quantitatives dont font état les auteurs.

II n'est donc pas exclu, comme ces derniers le suggèrent, que le tritium joue ici le rôle d'indicateur de la présence d'un autre agent toxique qui serait responsable de la corrélation statistiquement significative. Dans une déclaration dont a rendu compte la presse spécialisée [11] le Dr. Michael THORNE, secrétaire scientifique de la CIPR, a précisé que plusieurs des sujets morts du cancer de la prostate travaillaient dans des zones spécifiques des établissements de Harwell et de Winfrith où il $y$ avait des agents potentiellement carcinogènes autres que les rayonnements ionisants.

Pour toutes les raisons qui viennent d'être exposées, il est évident que des recherches doivent être poursuivies avant que l'on puisse porter un jugement définitif sur la cause de cet excès dans la mortalité par cancer de la prostate.

\subsubsection{Ensemble des résultats}

II est intéressant de rapprocher l'ensemble des résultats obtenus dans cette deuxième partie de ceux auxquels ont conduit précédemment des travaux analogues et plus particulièrement ceux de E.S. GILBERT et S. MARKS [8, 9] sur les employés de l'usine de Hanford. Aucune des affections malignes qui présentaient une corrélation significative avec l'exposition aux rayonnements à faibles doses dans les analyses antérieures, notamment : leucémie, myélome multiple, tumeurs du pancréas, du sein, du cerveau... ne montre ici de relation statistiquement significative avec les doses. La tendance est même négative pour les cancers du pancréas, du sein et du cerveau.

Réciproquement, le cancer de la prostate, qui est la seule affection maligne à présenter une corrélation significative dans l'étude menée sur les employés de I'UKAEA, n'apparaît avec de telles caractéristiques dans aucune des études antérieures; pour les employés de l'usine de Hanford, la tendance est même négative.

Ces faits sont suggestifs et plaident pour une certaine prudence dans l'interprétation de l'ensemble de tous ces résultats. Une corrélation positive statistiquement significative ne traduit pas nécessairement une relation de cause à effet et la non cohérence des résultats, comme celle qui apparaît ici, peut s'expliquer par la présence, dans les différents milieux de travail, d'agents carcinogènes divers (avec ou sans synergie avec les rayonnements) ou encore par l'occurence d'événements fortuits à probabilité faible comme le suggèrent DARBY, DOLL et PIKE [6].

\section{RELATION DOSE-RÉPONSE}

G. ROSE et coll. ont montré, dans la deuxième partie de leur publication, qu'il n'y avait pas de corrélation statistiquement significative entre l'exposition 
aux rayonnements et la mortalité par leucémie ou la mortalité par l'ensemble des cancers.

Cependant, les valeurs observées pour les 5 classes de dose définies pour cette étude font apparaître une tendance positive et il n'est pas illogique de rechercher, en tenant compte des incertitudes qui affectent chacune de ces valeurs. la relation linéaire qui traduit le mieux cette tendance. C'est ce qu'ont fait les auteurs dans la troisième partie de leur travail en partant, non pas des résultats de l'analyse interne, mais de ceux obtenus en prenant la population nationale comme population témoin et en ne tenant compte d'aucun temps de latence pour la mortalité par affection maligne dans la population étudiée, ce qui est peut-être critiquable.

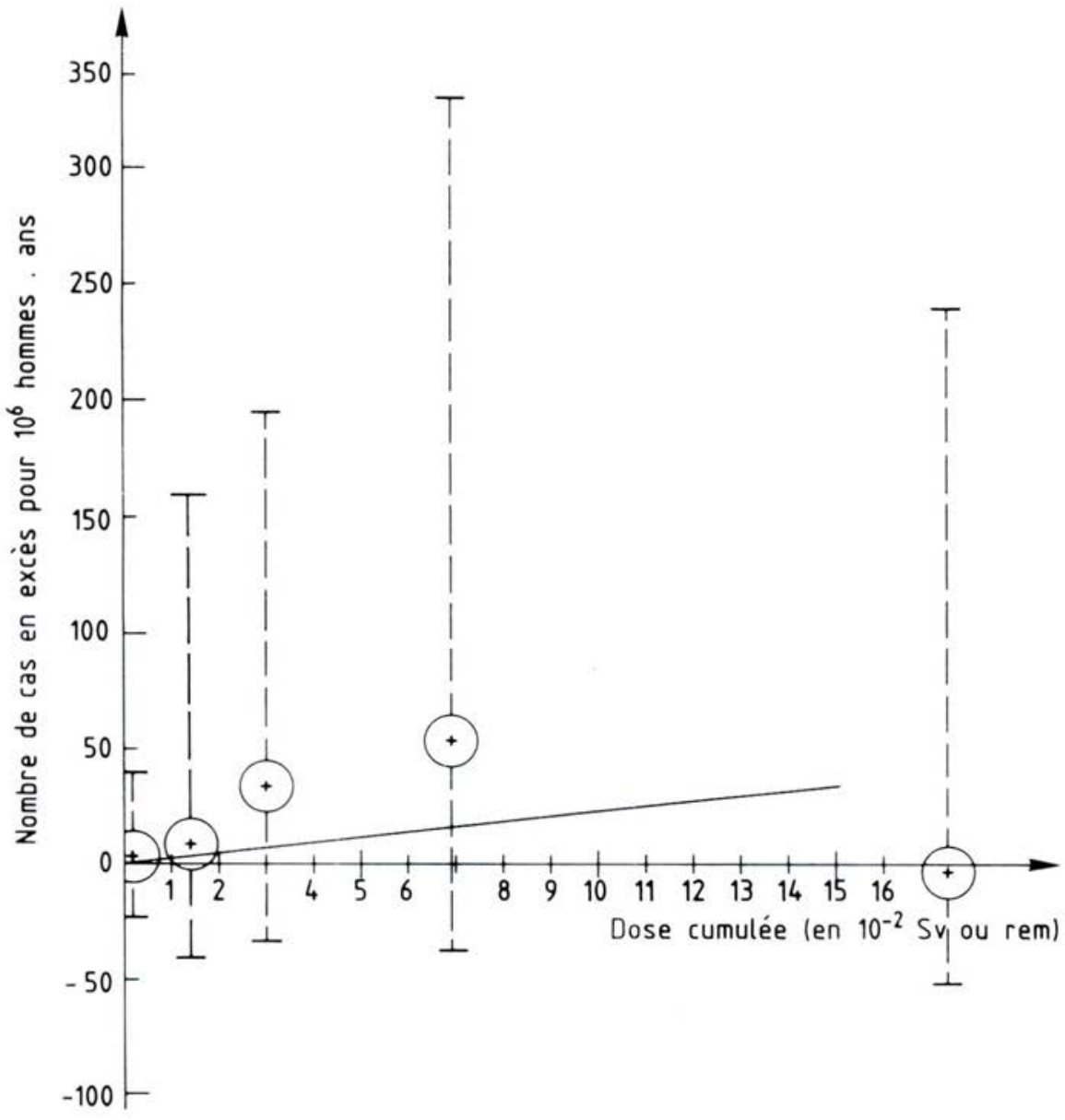

Fig. 1. - Mortalité par leucémie. Nombre de cas en excès pour $10^{6}$ hommes.an en fonction de la dose cumulée - Population témoin: Angleterre et Pays de Galles. Pente de la droite la plus probable: 2,2 cas par rem. 
Les données quantitatives pour chaque classe de dose ne figurent pas dans le document et le lecteur est obligé de s'en remettre aux deux représentations graphiques qui donnent, pour la mortalité par leucémie et pour la mortalité par l'ensemble des cancers, la différence (multipliée par $10^{6}$ ): nombre observé moins nombre attendu, divisée par le nombre de "personnes.an"(1) pour chaque classe de dose; on obtient ainsi l'excès de mortalité pour un million de personnes.an ou, si l'on veut, l'excès de décès par an pour un million de personnes (fig. 1 et 2 ).

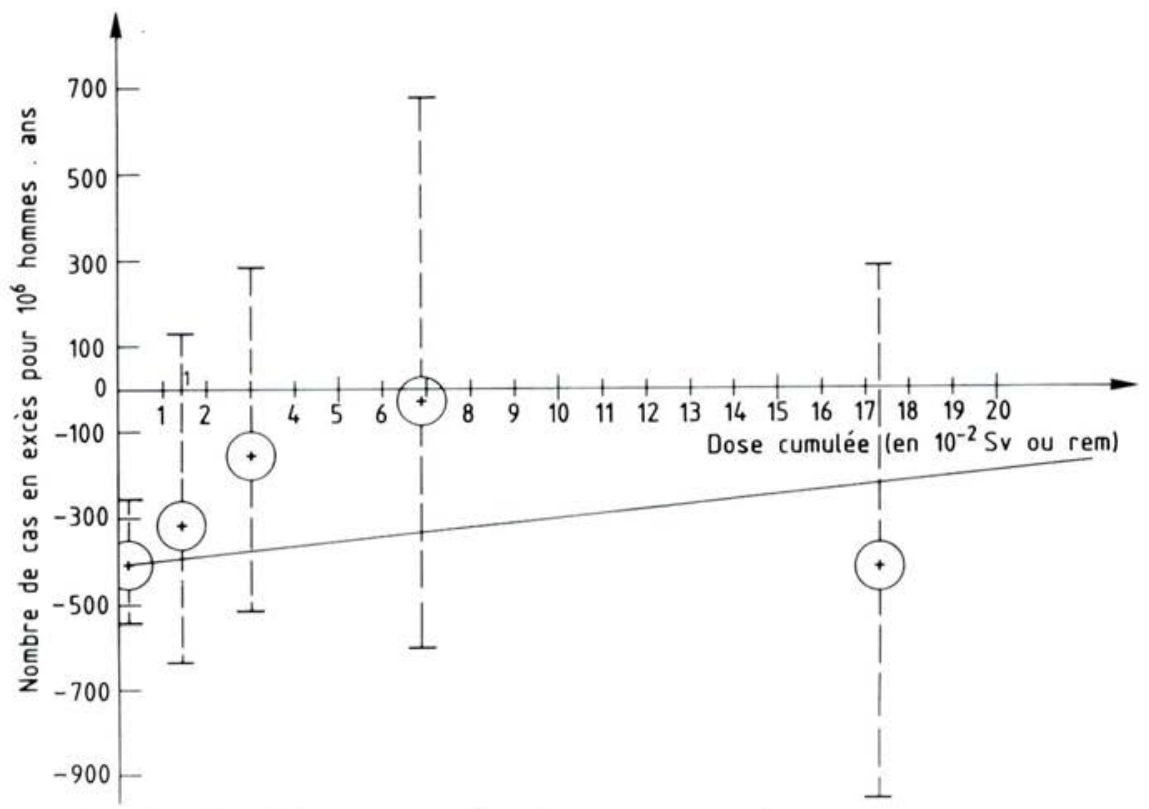

Fig. 2. - Mortalité par cancer. Nombre de cas en excès pour $10^{6}$ hommes.an en fonction de la dose cumulée - Population témoin: Angleterre et Pays de Galles.

Pente de la droite la plus probable: 12,5 cas par rem.

On peut remarquer que, pour la mortalité par leucémie, on trouve des valeurs positives sauf pour la dernière classe de dose $D \geqslant 0,1 \mathrm{~Sv}$ (10 rems). Pour la mortalité par cancer, toutes les valeurs sont négatives comme le laissait prévoir l'étude des SMR (tableau II) qui traduisait l'efficacité du "healthy working effect" même pour les affections malignes quand elles sont prises dans leur ensemble.

Ce qui est important pour juger de la valeur de ces résultats c'est la longueur des barres d'incertitude qui affectent chaque point représentatif. Ces marges d'incertitude sont considérables, ce qui résulte du caractère relativement limité en nombre de la population étudiée ainsi que de la durée moyenne de l'observation (16 ans) qui est assez courte par rapport à la vie humaine.

(1) Personnes.ans: chaque personne i qui a été observèe $t_{1}$ années contribue pour 1 personne multiplièe par $t_{i}$ ans, soit $1 \times t_{i}$ personnes.ans et pour les $n$ personnes observées dans la classe de dose considérée, la 
D'autre part, il est singulier que ce soit pour la classe d'exposition la plus élevée, $D \geqslant 0,1 \mathrm{~Sv}$ (10 rems), que l'excès présente la plus faible des 5 valeurs obtenues, aussi bien pour la leucémie que pour les cancers. Dans ces conditions, la pente des droites obtenues par régression ne peut qu'être affectée d'une considérable marge d'incertitude. Les auteurs ont ainsi trouvé comme pente la plus probable:

- pour la mortalité par leucémie $+2,2$ cas par an pour $10^{6}$ personnes recevant $0,01 \mathrm{~Sv}(1 \mathrm{rem})$ avec des limites de confiance $95 \%$ allant de $-2,7$ à $+12,4$;

- pour la mortalité par cancer

$+12,5$ cas par an pour $10^{6}$ personnes recevant $0,01 \mathrm{~Sv}$ ( 1 rem) avec des limites de confiance allant de $-22,0$ à $+52,2$.

On peut constater, comme le font les auteurs, que les pentes les plus probables ne diffèrent pas significativement de zéro; on peut même dire que des pentes légèrement négatives ne sont pas exclues. Les figures 3 et 4 représentent graphiquement ces marges d'incertitude ainsi que la droite correspondant au risque retenu par la CIPR. Si I'on admet que le risque-vie adopté par la CIPR $\left(20.10^{-6}\right)$ se répartit sur 25 ans, durée retenue par les auteurs, on trouve pour la mortalité par leucémie une pente de:

$+0,8$ cas par an pour $10^{6}$ personnes recevant $0,01 \mathrm{~Sv}(1$ rem)

et pour l'ensemble des cancers avec $100.10^{-6}$ sur la vie:

+4 cas par an pour $10^{6}$ personnes recevant $0,01 \mathrm{~Sv}$ ( 1 rem).

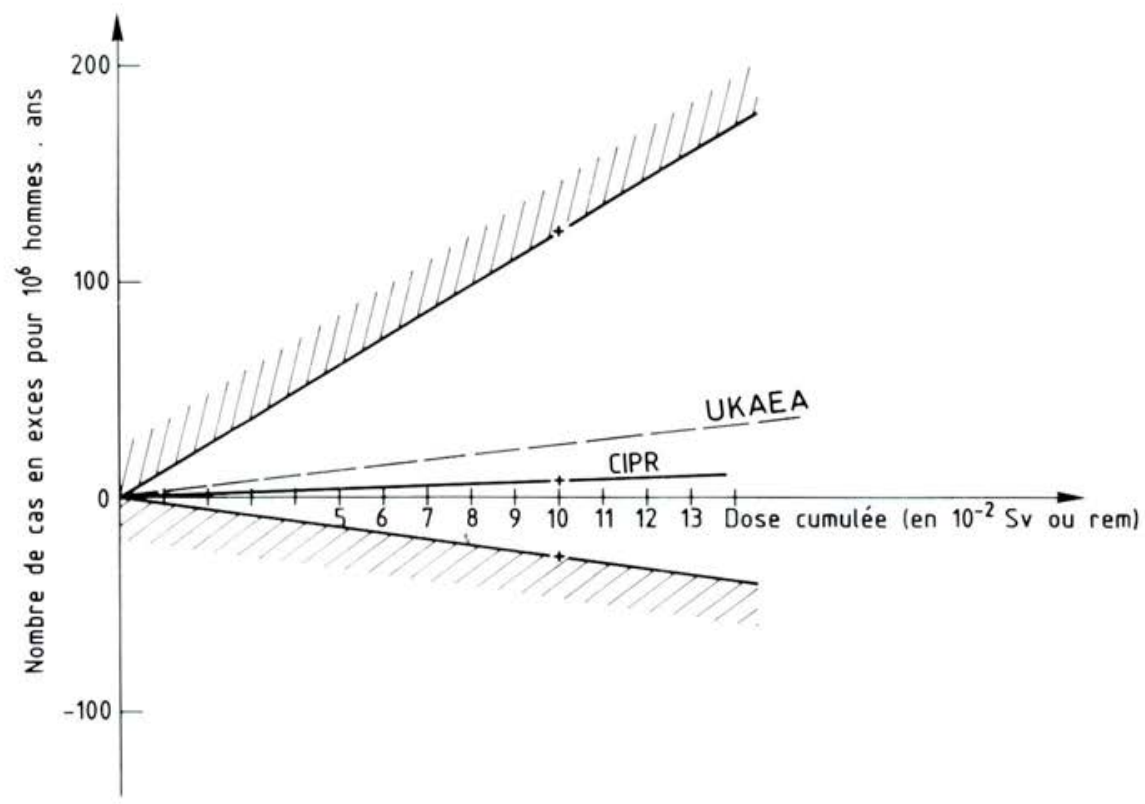

Fig. 3. - Mortalité par leucémie.

Marge d'incertitude $95 \%$ sur la pente de la relation linéaire dose-réponse. 


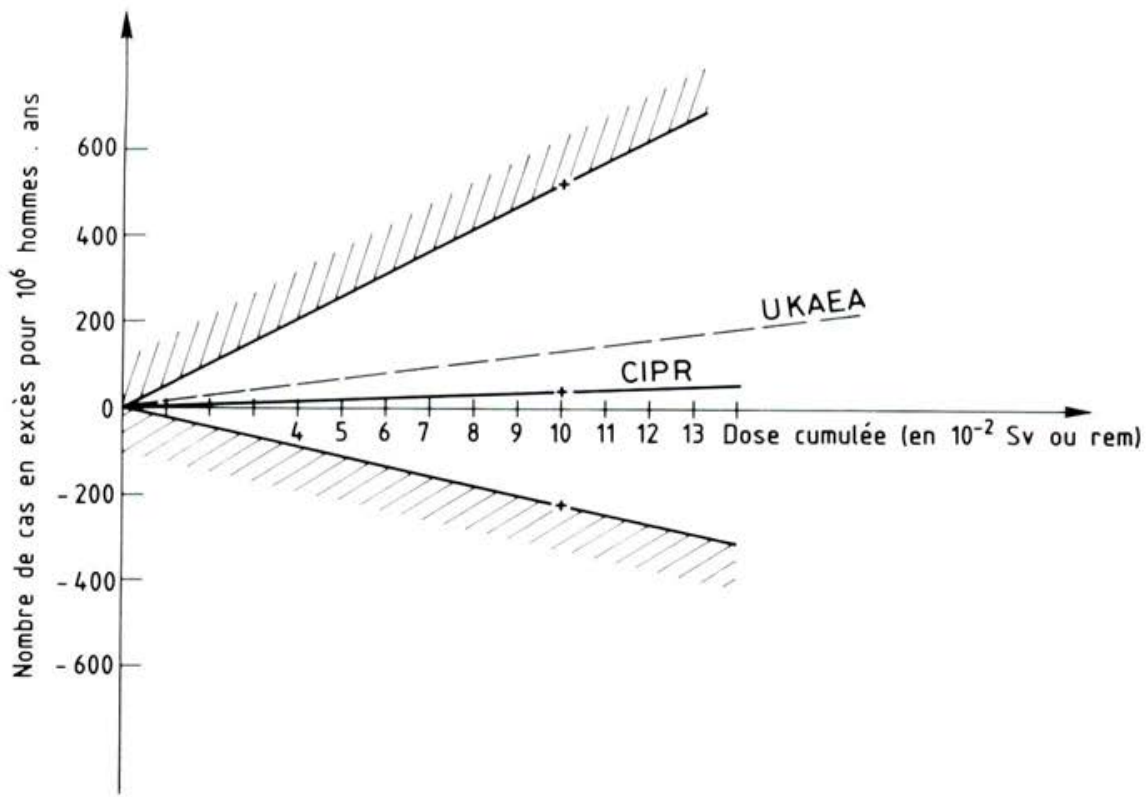

Fig. 4. - Mortalité par cancer.

Marge d'incertitude $95 \%$ sur la pente de la relation linéaire dose-réponse.

On voit qu'il y a un facteur 3 entre ces valeurs et les pentes les plus probables calculées avec les résultats de l'analyse statistique sur les employés de l'UKAEA et non un facteur 15 comme le déclarait le Times du 16 août 1985 en se référant, sans le dire, à la limite supérieure de la marge d'incertitude $95 \%$.

Dans une courte note citée plus haut [6], S.C. DARBY, R. DOLL et M.C. PIKE de I'Imperial cancer research fund, Oxford (G.B.) ont rendu compte d'une première tentative de combiner les résultats de cette enquête épidémiologique sur les employés de I'UKAEA avec ceux de l'enquête analogue menée par Gilbert et Marks $[8,9]$ sur la mortalité parmi les employés du Centre de Hanford. Cette synthèse suggère:

- pour la mortalité par leucémie

un excès de 1,3 cas par an pour $10^{6}$ personnes recevant $0,01 \mathrm{~Sv}$ ( $1 \mathrm{rem}$ );

- pour la mortalité par cancer

un excès de 6,0 cas par an pour $10^{6}$ personnes recevant $0,01 \mathrm{~Sv}$ ( 1 rem).

Le rapport avec les valeurs retenues par la CIPR n'est plus que 1,5.

Pour l'avenir, notons que l'étude sur le personnel de l'UKAEA se poursuit par l'exploitation des données des années 1980-1984. D'autre part, des recherches analogues ont été entreprises sur le personnel de British nuclear fuels (BNFL) et de Central electricity generating board (CEGB); elles devraient permettre un important regroupement d'informations statistiques susceptible de réduire dans des conditions satisfaisantes les marges d'incertitude par trop étendues qui affectent actuellement les résultats. 
Pour conclure, plutôt que de souligner, une fois de plus, la précipitation des médias pour annoncer une nouvelle à sensation fondée sur un travail scientifique un peu trop rapidement lu, il a paru plus opportun de compléter les pages qui précèdent par deux remarques.

1. La première concerne la fâcheuse habitude de dire et d'écrire que la CIPRa fondé ses recommandations sur une estimation du risque qui découle, par extrapolation aux faibles doses, des données obtenues à fortes doses sur les survivants de Hiroshima et de Nagasaki.

Indépendamment du fait que la CIPR disposait, en 1976, d'informations bien plus étendues que celles relatives aux survivants des 2 bombes nucléaires (expositions aux sources naturelles, expositions professionnelles, expositions médicales à des fins diagnostiques ou thérapeutiques, etc., sans oublier l'expérimentation animale) il faut se rappeler que l'échantillon le plus sérieusement étudié des survivants japonais ("Life span study") [10] comprenait sur 82242 sujets:

- 31581 personnes pour lesquelles la dose était tellement faible qu'on les a toutes regroupées dans la classe $D=0$;

- 23073 personnes pour lesquelles la dose était comprise entre 1 et 9 rads avec une moyenne de 3,4 rads;

- 14942 personnes pour lesquelles la dose était comprise entre 10 et 49 rads avec une moyenne de 21,8 rads.

II s'agit évidemment d'expositions "aiguës" mais non de "fortes doses"; l'éventail est, à quelque chose près, celui des doses cumulées dans les 2 études statistiques sur les employés de I'UKAEA et de Hanford.

2. La deuxième remarque a trait à la mortalité par cancer de la prostate. II n'est pas sans intérêt à ce sujet d'évoquer des chiffres extraits d'une étude en cours sur la mortalité par affection maligne dans la région du Limousin comparée à celle de la population du Poitou-Charentes prise comme population témoin. On sait [7] que les doses moyennes dues aux rayons $\gamma$ des sources naturelles de rayonnements diffèrent, pour ces deux régions, de $0,65 \mathrm{mSv}(65 \mathrm{mrem})$ par an (à 50 ans : 3,25 rems de plus pour l'habitant du Limousin, à 70 ans : 4,55 rems de plus) et que les populations masculines étaient, pour les années étudiées (1968 à 1975), d'environ 360000 pour le Limousin et 730000 pour le Poitou-Charentes. Pour les 8 années qui vont de 1968 à 1975, on trouve pour la mortalité par cancer de la prostate dans le Limousin: - nombre attendu (d'après les taux observés en Poitou-Charentes) ... 1027,81 - nombre observé ................................... 1029

Ainsi, en admettant que des doses cumulées de l'ordre de 3 à 5 rems aient un effet sur les décès par cancer de la prostate, cet effet est suffisamment faible pour être totalement noyé dans l'action de tous les paramètres qui, d'une façon générale. jouent un rôle dans l'étiologie de ce cancer.

\section{RÉFÉRENCES BIBLIOGRAPHIQUES}

[1] BEEBE G.W., KATO H., LAND C.E. Studies of the mortality of A-bomb survivors. 6 Mortality and radiation dose 1950-1974. Radiat. Res., 1978, 75, 138-201.

[2] BERAL V., INSKIP H., FRASER P., BOOT H.M., COLEMAN D., ROSE G. Mortality of employees of the United Kingdom Atomic energy authority, 1946-1979. Br. Med. J., 1985, $291,440-447$ 
[3] CHEKOWAY H., MATHEW R.M., WOLF S.H. et al. Mortality among workers at the Oak Ridge National Laboratory. In: Proceedings of the 16. midyear topical meeting of the Health physics society, 1983, CONF-830101, 90-104.

[4] COMMISSION INTERNATIONALE DE PROTECTION RADIOLOGIQUE (CIPR). Recommandations, Publication CIPR N ${ }^{\circ}$ 26. Oxford: Pergamon Press, 1980.

[5] COMMITTEE ON THE BIOLOGICAL EFFECTS OF IONIZING RADIATIONS, National research council. National academy of sciences (NAS-NRC). The effects on populations of exposure to low levels of ionizing radiations (BEIR). Washington: NAS, 1980.

[6] DARBY S.C., DOLL R., PIKE M.C. Mortality of employees of the UKAEA 1946-1979. Br. Med. J., 1985, 291, p. 672.

[7] DOUSSET M., JAMMET H. Comparaison de la mortalité par cancer dans le Limousin et le Poitou-Charentes. Radioprotection, 1985, 20 (1), 61-67.

[8] GILBERT E.S., MARKS S. An analysis of the mortality of workers in a nuclear facility. Radiat. Res., 1979, 79, 122-148.

[9] GILBERT E.S., MARKS S. An updated analysis of the mortality of workers in a nuclear facility. Radiat. Res. 1980, 83, 740-741.

[10] KATO H., SCHULL W.J. Studies of the mortality of A-bomb survivors. 7 - Mortality 1950-1978. Part I - Cancer mortality. Radiat. Res., 1982, 90, 395-432.

[11] The MRC study and ICRP limits. Nucl. Eng. Int., 1985, 20 (375), p. 7.

[12] SMITH P.G., DOLL R. Mortality among patients with ankylosing spondylitis after a single treatment course with $\mathrm{X}$ rays. Br. Med. J., 1982, 284, 449-460. 